\section{Proteolysis targeting chimeric molecules as therapy for multiple myeloma: efficacy, biomarker and drug combinations}

\author{
Su Lin Lim, ${ }^{1 *}$ Alisa Damnernsawad, ${ }^{2 *}$ Pavithra Shyamsunder, ${ }^{3}$ Wee Joo Chng, ${ }^{3}$ \\ Bing Chen Han, ${ }^{1}$ Liang $\mathrm{Xu},{ }^{3}$ Jian Pan, ${ }^{1}$ Dakle Pushkar Pravin, ${ }^{3}$ Serhan Alkan, ${ }^{1}$ \\ Jeffrey W. Tyner ${ }^{2 * *}$ H. Phillip Koeffler ${ }^{1,3^{* *}}$ \\ ${ }^{1}$ Cedars Sinai Medical Center, Los Angeles, CA, USA; ${ }^{2}$ Division of Hematology and Medical \\ Oncology, Oregon Health and Science University Knight Cancer Institute, Portland, OR, \\ USA and ${ }^{3}$ Cancer Science Institute of Singapore, National University of Singapore, \\ Singapore
}

*SLL and AD contributed equally to this work. **JWT and HPK contributed equally to this work
Haematologica 2019
Volume 104(6):1209-1220
P roteolysis targeting chimeric molecule ARV 825 causes ubiquitination of bromodomains resulting in their efficient degradation by proteasome activity. Bromodomain degradation down-regulates MYC transcription contributing to growth inhibition of various human cancers. We examined the therapeutic potential of ARV 825 against multiple myeloma (MM) cells both in vitro and in vivo. In a dose-dependent manner, ARV 825 inhibited proliferation of 13 human $\mathrm{MM}$ cell lines and three fresh patient samples, and was associated with cell cycle arrest and apoptosis. ARV 825 rapidly and efficiently degraded BRD 2 and BRD 4. Sensitivity of MM cells to ARV 825 was positively correlated with cereblon levels. RNA sequencing analysis showed important genes such as CCR1, RGS, MYB and MYC were down-regulated by ARV 825. A total of 170 small molecule inhibitors were screened for synergy with ARV 825. Combination of ARV 825 with inhibitor of either dual PI3K/mTOR, CRM1, VEGFR, PDGFR $\alpha / \beta$, FLT3, IGF-1R, protein kinase C, CBP-EP300 or JAK1/2 showed synergistic activity. Importantly, ARV 825 significantly inhibited the growth of MM xenografts and improved mice survival. Taken together, our results, in conjunction with recently published findings, provide a rationale for investigating the efficacy of ARV 825 for MM, use of cereblon as a biomarker for therapy of MM patients, and the combination of ARV 825 with small molecule inhibitors to improve the outcome of MM patients.

\section{Introduction}

Multiple myeloma (MM) is characterized by neoplastic proliferation of clonal plasma cells producing a monoclonal immunoglobulin. It accounts for more than $17 \%$ of hematologic malignancies in the US. ${ }^{1}$ Over the past decade, newly introduced therapeutic regimens (e.g. proteasome inhibitor and immunomodulatory drugs) have significantly improved treatment outcome and survival of MM. Nevertheless, most of these patients eventually relapse, underlining the need for new therapeutic approaches. Agents with novel mechanism of action such as monoclonal antibodies (e.g. daratumumab, elotuzumab), histone deacetylase inhibitors, kinesin spindle protein inhibitors, and cereblon modulator iberdomide are under ongoing investigation for treating $M M$. Other than that, chimeric antigen receptor $\mathrm{T}$ (CAR-T) cells directed against $\mathrm{B}$-cell maturation antigen (BCMA) have shown promising tumor cell reduction in $\mathrm{MM} .{ }^{2}$ The search for novel agents is rapidly expanding and this, together with identification of novel combinations, should help revolutionize treatment of this disease.

Bromodomains (BRD) 2, 3 and 4, and $\mathrm{T}$ are members of the bromodomain extraterminal domain (BET) family facilitating transcriptional activation by RNA polymerase II. ${ }^{3}$ BRD 2, 3 and 4 bind to acetylated chromatin promoting progression from G1 to

\section{Correspondence:}

SU LIN LIM

sulin_lim_86@hotmail.com

Received: July 11, 2018.

Accepted: January 2, 2019.

Pre-published: January 3, 2019.

doi:10.3324/haematol.2018.201483

Check the online version for the most updated information on this article, online supplements, and information on authorship \& disclosures: www.haematologica.org/content/104/6/1209

(C)2019 Ferrata Storti Foundation

Material published in Haematologica is covered by copyright. All rights are reserved to the Ferrata Storti Foundation. Use of published material is allowed under the following terms and conditions:

https://creativecommons.org/licenses/by-nc/4.0/legalcode. Copies of published material are allowed for personal or internal use. Sharing published material for non-commercial purposes is subject to the following conditions:

https://creativecommons. org//icenses/by-nc/4.0/legalcode, sect. 3. Reproducing and sharing published material for commercial purposes is not allowed without permission in writing from the publisher. 
S phase of the cell cycle by direct interaction with positive transcription elongation factor complex b. ${ }^{4} \mathrm{BRD} 4$ is often located in super-enhancer regions associated with key genes (e.g. MYC, IGLL5, IRF4, PRDM1/BLIMP-1, and $X B P 1)$. These super-enhancer driven genes are also important in MM biology, playing key roles in controlling cell proliferation. $^{5}$

The BET inhibitor JQ1 has potent anti-MM activity in vitro and in vivo, ${ }^{6}$ but its reversible binding to BRD proteins causes incomplete transcriptional repression of MYC and other oncoproteins. ${ }^{7}$ It also does not induce apoptosis in MM. ARV 825 (Arvinas Inc., New Haven, CT, USA) is a hetero-bifunctional molecule composed of a BRD 4 binding moiety (OTX015) joined to pomalidomide. The latter binds to an E3 ubiquitin ligase, cereblon (CRBN) and OTX 015 brings the complex to the BRD molecules. These drugs are called PROTAC (proteolysis targeting chimeric molecules) causing ubiquitination of $\mathrm{BRD}$, resulting in rapid and efficient degradation by proteasome activity. 9,10 PROTAC have potent activity against lymphoma, leukemia, and prostate cancers. ${ }^{7,9-11}$ Their activity on myeloma models has also been described. dBET1 (composed of JQ1 joined to thalidomide) promoted degradation of BRD 4 in an MM cell line (MM1S) ${ }^{12}$ and a recent publication showed that BET targeted PROTAC (ARV 825 and ARV 763) has anti-myeloma activity associated with decreasing MYC and Akt/mTOR. ${ }^{13}$ The authors also showed that ARV 825 was active against primary myeloma cells both in vitro and in vivo, and could overcome drug resistance in $\mathrm{MM}$ cells. ${ }^{13}$

In this study, we demonstrate that ARV 825 (8.5-500 $\mathrm{nM}$ ) inhibited cell proliferation of 13 human MM cell lines and three fresh myeloma samples in vitro. In addition, the drug induced apoptosis, cell cycle arrest in vitro, and had growth inhibitory activity against MM cells in vivo. This PROTAC inhibited growth of MM cells resistant to either glucocorticoids or bortezomib, as well as those with t(4:14) translocation and FGFR3 and MMSET overexpression (poor prognosis). We identified prominent levels of CRBN as a biomarker of responsiveness to the drug. Those MM cells resistant to ARV 825 were sensitive to another PROTAC (MZ1) relying on a different E3 ligase (VHL). We also examined 170 drugs [US Food and Drug Administration (FDA)-approved or in clinical trial] for their ability to enhance the cell inhibitory activity of ARV 825. In depth analysis showed synergy of ARV 825 with either LY3023414 (dual PI3K/mTOR inhibitors), selinexor (CRM1 inhibitor), cediranib (VEGFR inhibitor), crenolanib (PDGFR $\alpha / \beta$ and FLT3 inhibitor), GSK 1904529A (IGF-1R inhibitor), motesanib (VEGFR1/2/3 inhibitor), gilteritinib (FLT3/AXL inhibitor), LY333531 (protein kinase C inhibitor), IGC003 (CBP-EP300 inhibitor), or ruxolitinib (JAK1/2 inhibitor).

\section{Methods}

\section{Cell culture}

All cell lines were cultured and maintained in RPMI1640 containing $10 \%$ fetal bovine serum (FBS) and 1\% penicillin-streptomycin (Invitrogen, Carslbad, CA, USA) at $37^{\circ} \mathrm{C}$ with $5 \% \mathrm{CO}_{2}$. The 8226 LR5 cells were maintained in $10 \mathrm{nM}$ melphalan, the 8226 P100V cells were cultured with $100 \mathrm{nM}$ bortezomib for two days every two weeks. Short random repeat (STR) analysis was carried out on all cell lines used in this study.

\section{Cell Titer-Glo luminescent cell viability assay}

Three primary MM patient samples obtained from their bone marrow (BM) were plated in 96-well plates with different concentrations of ARV 825 [dimethyl sulfoxide (DMSO) as a vehicle]. After 48 hours (h), cell viability was determined using the CellTiter-Glo® luminescent cell viability kit according to the manufacturer's instructions. The luminescence was measured by a luminometer (GloMax®-Multi Detection System Madison, WI, USA). All experiments were repeated at least three times. The means with standard deviations were shown.

\section{Lentivirus production, gene knockdown and overexpression of CRBN}

shRNA targeting CRBN in pLKO.1 lentiviral vector (Sequence: CCGGGCCCACGAATAGTTGTCATTTCTCGAGAAATGACAACTATTCGTGGGCTTTTTG) and pLX304-CRBN-V5 vector (PMID: 29764999) were a kind gift from Dr. X. Liang (Cancer Science Institute, Singapore). Luciferase vector was purchased from Addgene (plasmid \#17477). Recombinant lentiviral vector and packaging vector (pCMV-dR8.9 and pMD2.G-VSVG) were co-transfected into $293 \mathrm{FT}$ cells using polyethylenimine (PEI) according to the manufacturer's instructions. Virus supernatants were harvested at $48 \mathrm{~h}$ and $72 \mathrm{~h}$ after transfection, and placed through a $0.45 \mu \mathrm{m}$ filter. KMS11 and KMS28BM cells $\left(1 \times 10^{6}\right.$ per well) were seeded in 6-well plates. Cells were transduced with lentivirus vectors in the presence of $8 \mu \mathrm{g} / \mathrm{mL}$ polybrene (SigmaAldrich) for $24 \mathrm{~h}$. Stable cell lines were selected with either puromycin or blasticidin.

\section{In vivo xenografts}

To access the in vivo activity of ARV 825, KMS11 expressing luciferase (KMS11LUC) were injected into the lateral tail vein of SCID-Beige mice $(n=9)$ versus diluent control mice $(n=9)$. Mice were monitored for 14 days and images were taken with a Xenogen IVIS spectrum camera (PerkinElmer, MA, USA) to document engraftment before treatment was initiated. After 14 days, mice were treated with either vehicle alone $\left(5 \%\right.$ Kolliphor ${ }^{\circledR}$ HS15) or $5 \mathrm{mg} / \mathrm{kg}$ of ARV 825 (daily intraperitoneal injection). Tumor burden in each treatment group was photographed weekly with a Xenogen camera and the overall survival (OS) monitored.

\section{Statistical analysis}

For in vitro and in vivo experiments, the statistical significance of difference between two groups used two-tailed Student $t$-test and two-way ANOVA. Significant differences between experimental groups in comparison to controls are shown: ${ }^{*} P<0.01 ;{ }^{* *} P<0.001$; ${ }^{* * *} P<0.0001$. Means \pm Standard Deviation (SD) are shown.

All animal care and experimental procedures in this study complied with the protocol approved by the Institutional Animal Care and Use Committee at Cedars Sinai Medical Center, Los Angeles, CA, USA.

For detailed information on the materials and methods used see the Online Supplementary Appendix.

\section{Results}

ARV 825 significantly inhibits cellular proliferation and clonogenic growth of multiple myeloma cells

Structures of ARV 825 and MZ1 are shown in Figure 1A. The ARV 825 is composed of the BET inhibitor OTX 015 conjugated to the ligand for cereblon E3 ligase. Another PROTAC (MZ1) is composed of the BET inhibitor JQ1 conjugated to the ligand for VHL E3 ligase. ARV 825 was tested in a dose-dependent manner against 
A

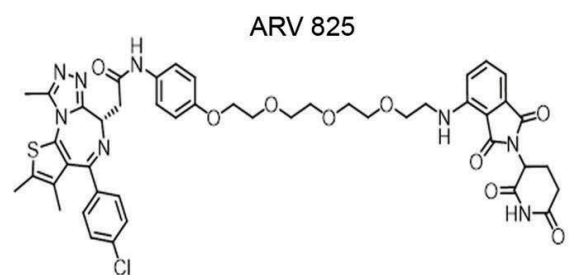

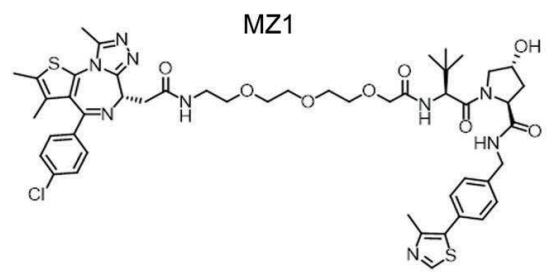

OTX015

PEG linker Pomalidomide

JQ1

PEG linker

VHL ligand

B

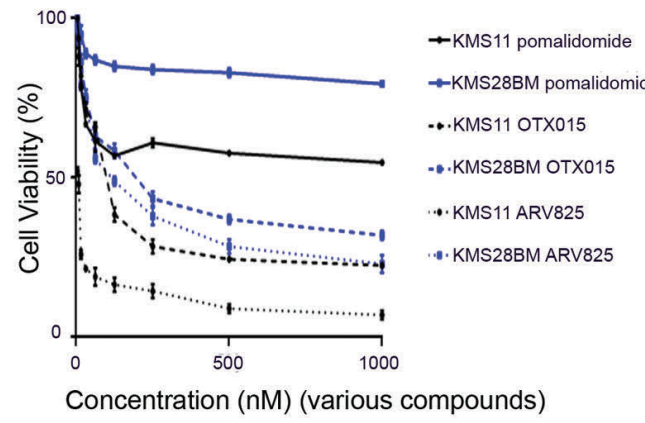

D

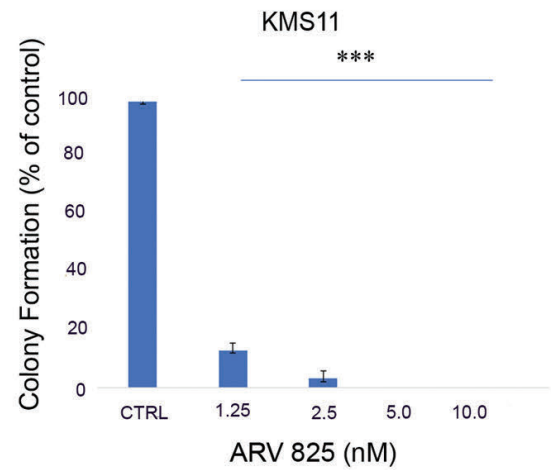

E

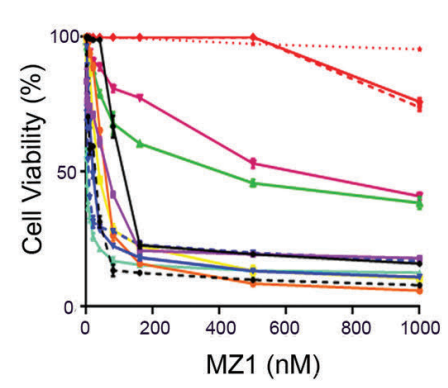

$\rightarrow$ H929

$\rightarrow$ KMS11

-..KMS11 res

$\rightarrow \mathrm{MM} 1 \mathrm{~S}$

-.MM1S res

$\rightarrow$ KMS28BM

$\rightarrow$ KMS12BM

-MM1R

- U266

$\rightarrow$ KMS18

$\rightarrow 8226$ LR5

-.. 8226

... $8226 \mathrm{P} 100 \mathrm{~V}$
C

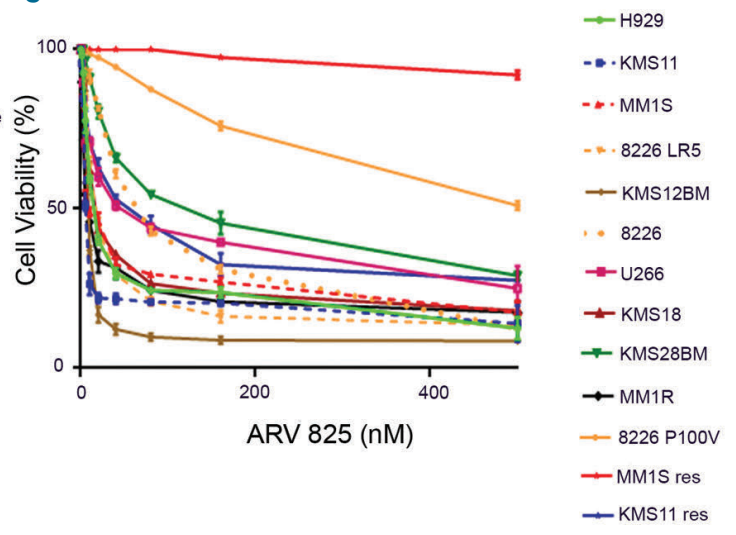

KMS28BM
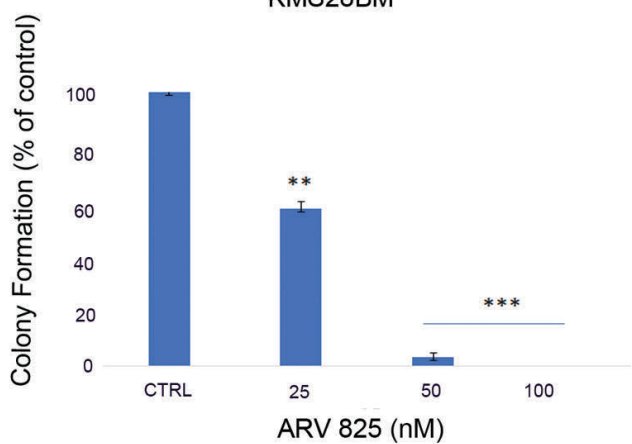

F

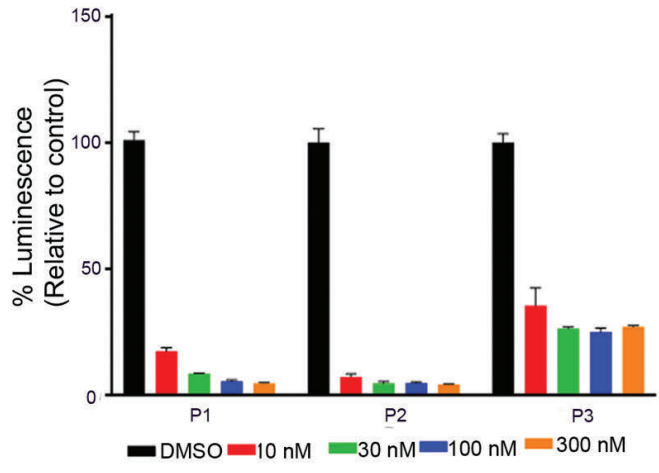

Figure 1. ARV 825 anti-proliferative activities: MTT and clonogenic growth of multiple myeloma (MM) cells. (A) Structures of the two proteolysis targeting chimeric molecules (PROTAC) used in this study: ARV 825 and MZ1. (B) Growth inhibition of KMS11 and KMS28BM cells treated with pomalidomide ( $1 \mu M-20 \mu M)$, OTX015 $(1 \mu \mathrm{M}-10 \mu \mathrm{M})$, and ARV $825(1 \mathrm{nM}-1000 \mathrm{nM})$ for 72 hours $(\mathrm{h})$. Results are Mean \pm Standard Deviation (SD); three experiments were carried out in triplicate. (C) MM cells were treated with ARV 825 ( 1 nM-500 nM, 72 h). Growth inhibition was measured by MTT assay. Results are Mean \pm SD; n=3. IC50s are shown in Online Supplementary Table S1. (D) ARV 825 decreased clonogenic growth of KMS11 and KMS28BM cells. Mean \pm SD of two independent experiments carried out in triplicates. Student $t$-test, $* * P \leq 0.001 ; * * * P \leq 0.0001$. (E) MM cells treated with PROTAC MZ1 (VHL E3 ligase fused to JQ1; 1-1000 nM, 72 h, and measured by MTT assay). Results represent Mean \pm SD of three experiments carried out in triplicate. IC50s are shown in Online Supplementary Table S2. (F) Primary patient samples (Patient 1, Patient 2, Patient 3) treated with ARV 825 (10-300 nM, 72 h). Growth inhibition measured by luminescence cell viability assay. Results are Mean \pm SD; $\mathrm{n}=3$. 
A
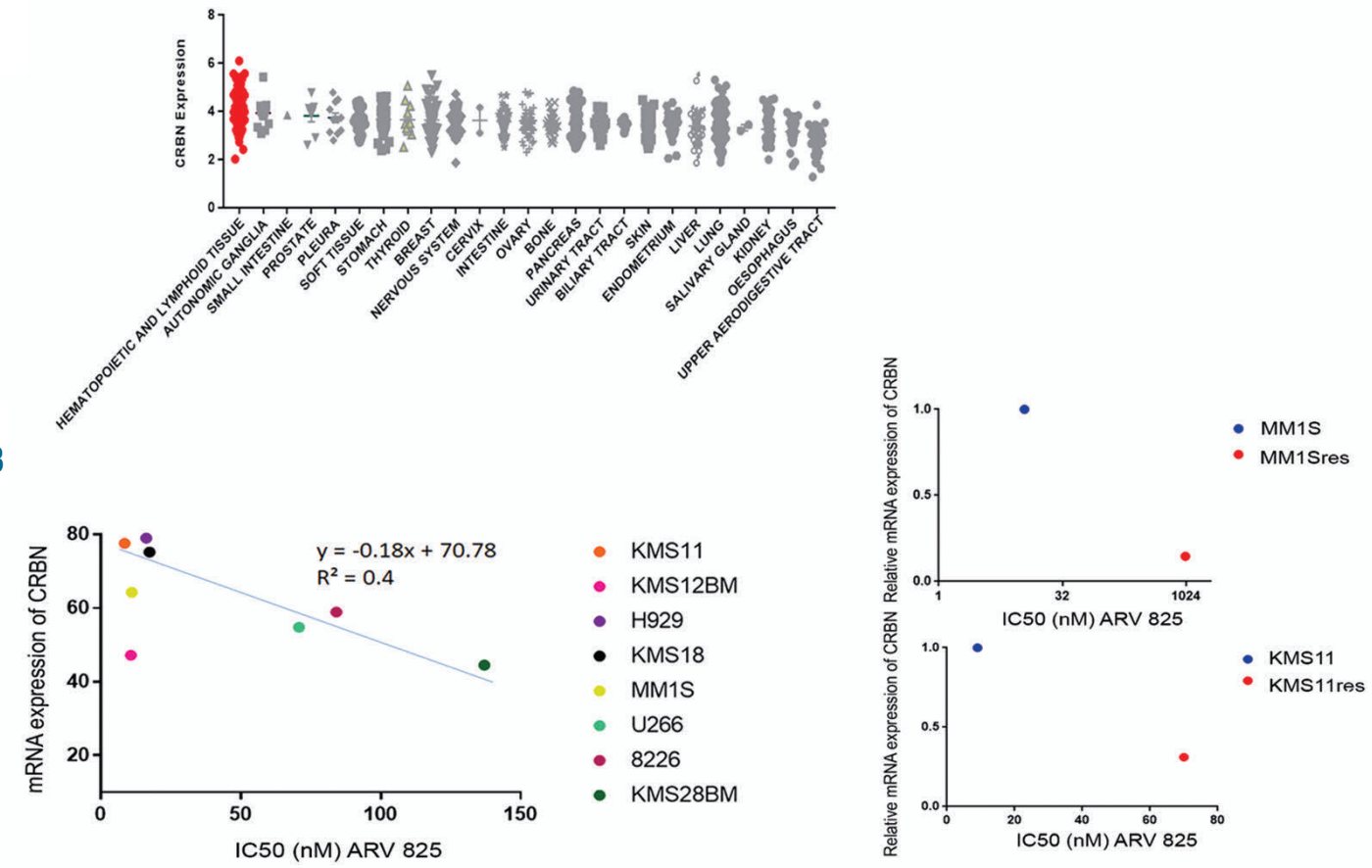

C
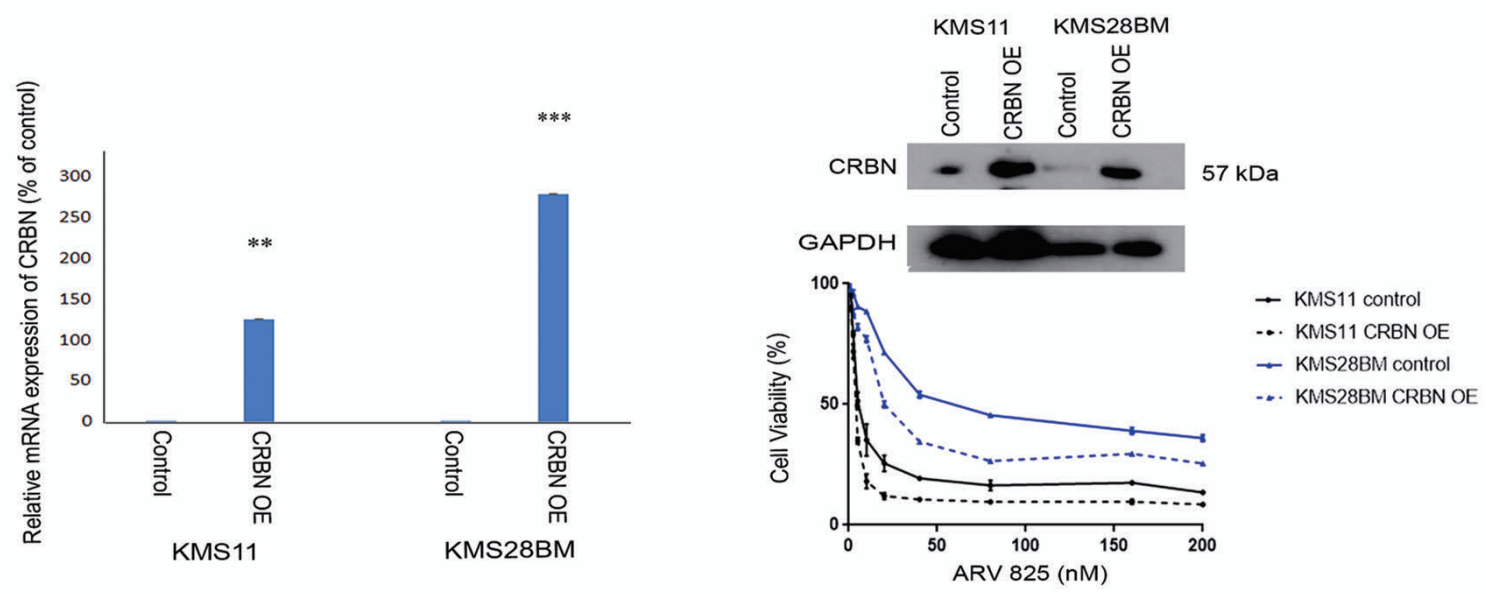

D
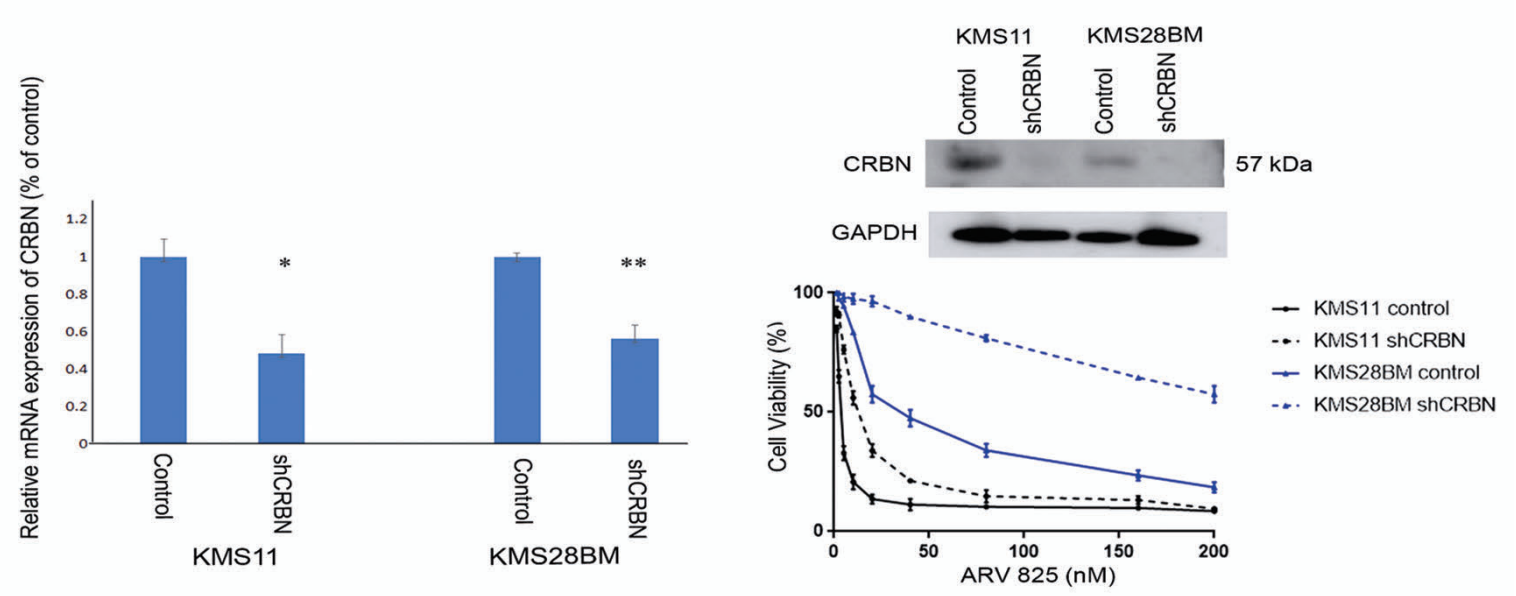

Figure 2. CRBN mRNA levels of multiple myeloma (MM) cells and their correlation with sensitivity to ARV 825. (A) CRBN expression in tumor cell lines (Broad Cancer Cell Line Encyclopedia). (B) Positive correlation [correlation coefficient $(R)=0.6$ ] between CRBN mRNA expression in eight parental MM cell lines $\left(2-{ }^{\Delta(c)} \times 10^{5}\right)$, and their sensitivity to ARV 825, $\mathrm{n}=3$; $P<0.001$ ) (Left). Relative CRBN mRNA expression of isogenic lenalidomide resistant (MM1S res and KMS11 res) and isogenic wildtype (MM1S and KMS11) myeloma cells, and comparison of their sensitivity to increasing concentrations of ARV 825 [growth inhibition (MTT) of the cells]. (Right, top) MM1S and MM1S res cells and (right, bottom) shows KMS11 and KMS11 res cells, and their IC50s to ARV 825. (C and D) Levels of CRBN mRNA expression (left) and protein (right, top) from KMS11 and KMS28BM cells, and their viability in ARV 825 (right, bottom) after (C) overexpression and (D) shRNA silencing. OE: overexpression. ${ }^{*} P \leq 0.01 ; * * P \leq 0.001 ; * * * P \leq 0.0001$. 
A

KMS11

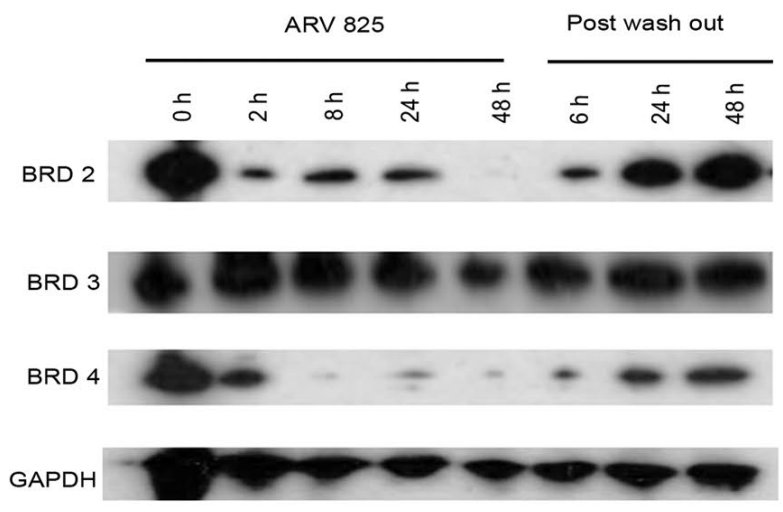

KMS11

B

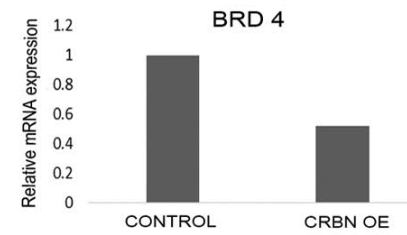

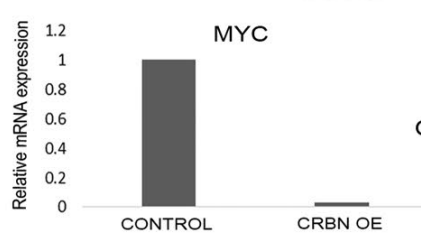

KMS11

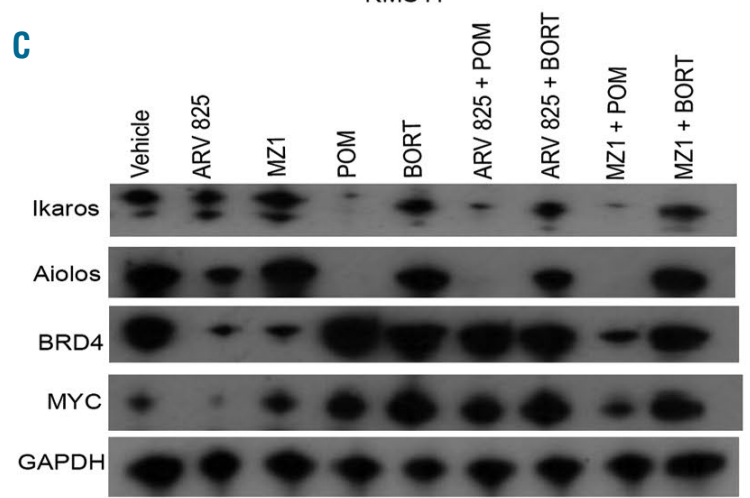

KMS28BM

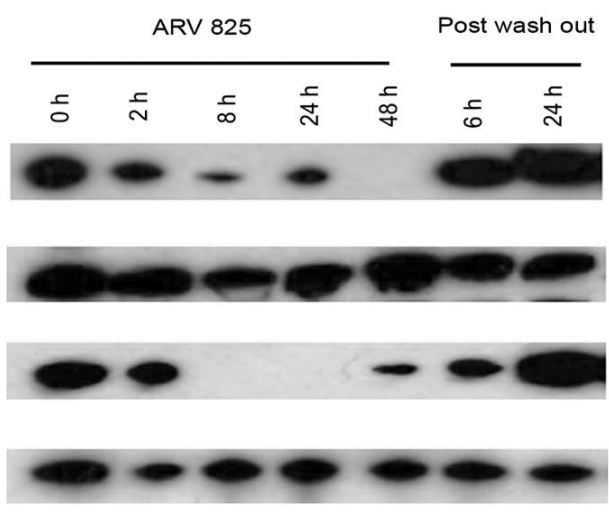

BRD 4

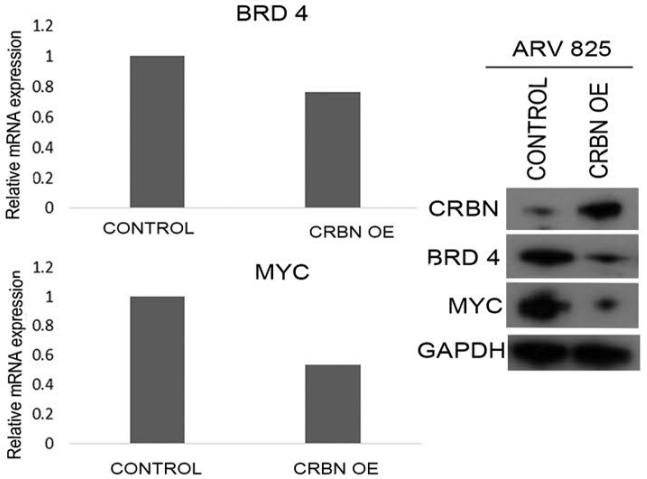

KMS28BM

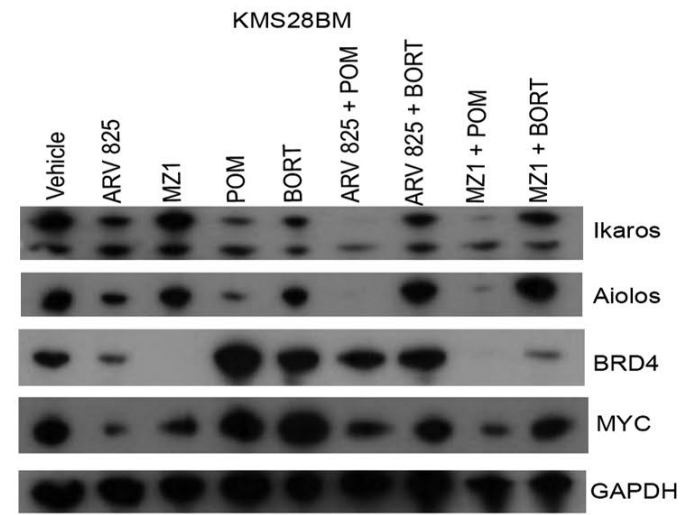

D Combined effect of Proteosome Inhibitor (Bortezomib) with ARV 825

Combined effect of Pomalidomide with ARV 825

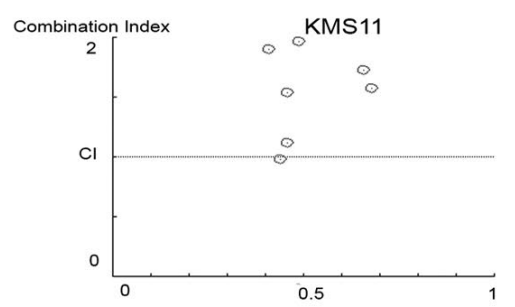

Figure 3. ARV 825 rapidly degrades BRD 2 and BRD 4 in KMS11 and KMS28BM multiple myeloma (MM) cells. (A) Immunoblotting of BRD 2 , BRD 3 and BRD 4 in KMS11 and KMS28BM cells, cultured for different times with ARV 825: $10 \mathrm{nM}$ (KMS11) and 100 nM (KMS28BM). Glyceraldehyde-3-phosphate dehydrogenase (GAPDH): internal control. (B) Levels of BRD 4 and MYC mRNA expression (left, top and bottom) and protein expression (right) using wild-type KMS11, KMS28BM and their respective cells over-expressing CRBN, cultured with either $10 \mathrm{nM}$ (KMS11) or $100 \mathrm{nM}$ (KMS28BM) of ARV 825 for $4 \mathrm{~h}$. GAPDH: internal control. (C) Protein expression of IKZF 1/3 (Ikaros/Aiolos), BRD 4 and MYC after treatment with ARV 825 (10 nM, KMS11; 100 nM, KMS28BM), MZ1 (100 nM, KMS11; 70 nM, KMS28BM), or combinations of the two PROTAC with either $10 \mu \mathrm{M}$ pomalidomide (POM) or $5 \mathrm{nM}$ bortezomib (BORT) for $12 \mathrm{~h}$ and examined by immunoblotting (GAPDH: internal control). (D) Combination Index plot of ARV 825 with either bortezomib or pomalidomide on KMS11 cells. 
a panel of 13 human MM cell lines (KMS11, MM1R, KMS12BM, H929, KMS18, 8226 LR5, MM1S, KMS11 res, U266, 8226, KMS28BM, 8226 P100V, MM1S res) using an in vitro proliferation assay (MTT, $72 \mathrm{~h}$ ). Cell lines included melphalan resistant (8226 LR5), steroid resistant (MM1R), bortezomib resistant (8226 P100V), and lenalidomide resistant (KMS11 res and MM1S res) cell lines. Their cytogenetics varied; some were associated with a poor prognosis [e.g. t(4:14): KMS11, KMS28BM, H929; t(14:16): MM1S, 8226. ARV 825 was more potent than either OTX 015 or pomalidomide alone against both KMS11 (IC50 for ARV 825, OTX 015 and pomalidomide: $9 \mathrm{nM}, 130 \mathrm{nM}$, $>1000 \mathrm{nM}$, respectively) and KMS28BM cells (IC50 for ARV 825, OTX 015 and pomalidomide: $137 \mathrm{nM}, 240 \mathrm{nM}$, $>1000 \mathrm{nM}$, respectively) (Figure 1B). All MM cell lines were sensitive to ARV 825 with an IC50 ranging from 8 $\mathrm{nM}$ to $500 \mathrm{nM}$ except for MM1S res cells (>1000 nM) (Figure 1C). MM1S res cells (resistant to lenalidomide) had significantly reduced CRBN levels (Online Supplementary Figure $S 1 A$ and $B$ ). The MM1S res cells had a 40 -fold reduction in expression of CRBN compared to parental MM1S cell line due to deletion of one allele of the CRBN gene and a point mutation on the second allele. ${ }^{14}$ Therefore, because of lack of wild-type CRBN, they had loss of wild-type CRBN expression (western blot, Online Supplementary Figure $S 1 B$ ) associated with a resistance to ARV 825. Likewise, KMS11 res cells (resistant to lenalidomide) also have a structural deletion of CRBN with reduced CRBN expression ${ }^{15}$ (Online Supplementary Figure S1A and B) and were 8.3-fold more resistant to ARV 825 compared to its parental cells. Overexpression of wild-type CRBN in MM1S res cells rescued this resistant cell line and increased its sensitivity to ARV 825 (IC50=800 nM) (Online Supplementary Figure S1C). KMS11 cells are the most sensitive, with an IC50 of $8.5 \mathrm{nM}$; in contrast, KMS28BM is a relatively more resistant cell line (IC50=137 nM) (Online Supplementary Table S1). ARV 825 decreased clonogenic growth of KMS11 and KMS28BM in a dose-dependent manner (Figure 1D).

The other PROTAC (MZ1) significantly suppressed growth of the lenalidomide resistant cells (MM1S res and KMS11 res) as well as some of the other MM cells. However, KMS18, U266, 8226, 8226 LR5 and 8226 P100V were relatively resistant to MZ1 (Figure 1E). IC50s are shown in Online Supplementary Table S2.

The bortezomib-resistant cell line (8226 P100V) is also relatively resistant to ARV 825 (IC50=500 nM). Consistently, the BRD 4 degradation in $8226 \mathrm{P} 100 \mathrm{~V}$ cells after treatment with different doses of ARV $825(50 \mathrm{nM}$, $100 \mathrm{nM}, 200 \mathrm{nM})$ only modestly decreased when compared to its parental strain (8226). $8226 \mathrm{P} 100 \mathrm{~V}$ has up-regulated mRNA expression of multidrug resistance-associated protein 1 (MRP-1, encoded by the ABCC1 gene) compared to its parental strain (8226) (Online Supplementary Figure S1D).

\section{ARV 825 inhibit growth of primary myeloma cell samples}

The effect of ARV 825 (10 nM to $300 \mathrm{nM}$ ) on three MM patient samples was evaluated. The PROTAC significantly inhibited growth of these MM patient samples which include one multiple relapsed patient (Patient 3). $10 \mathrm{nM}$ of ARV 825 inhibited the growth of MM Patients 1, 2 and 3 by $82 \%, 93 \%$, and $64 \%$, respectively (Figure $1 \mathrm{~F}$ ).
A

KMS11

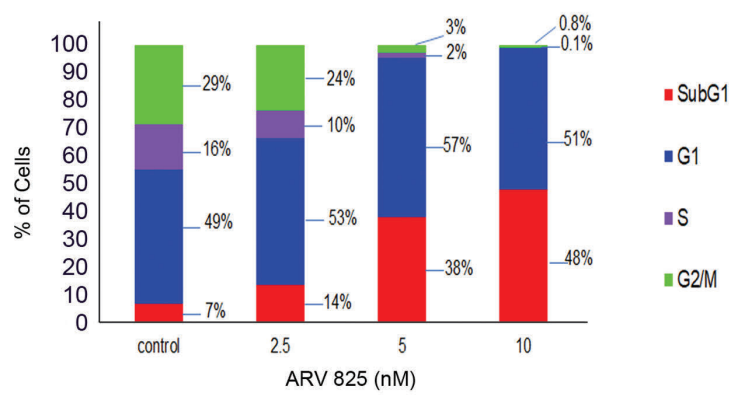

KMS28BM

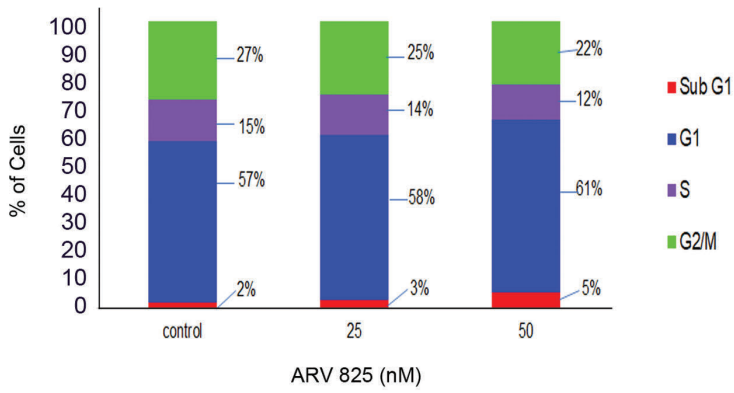

B
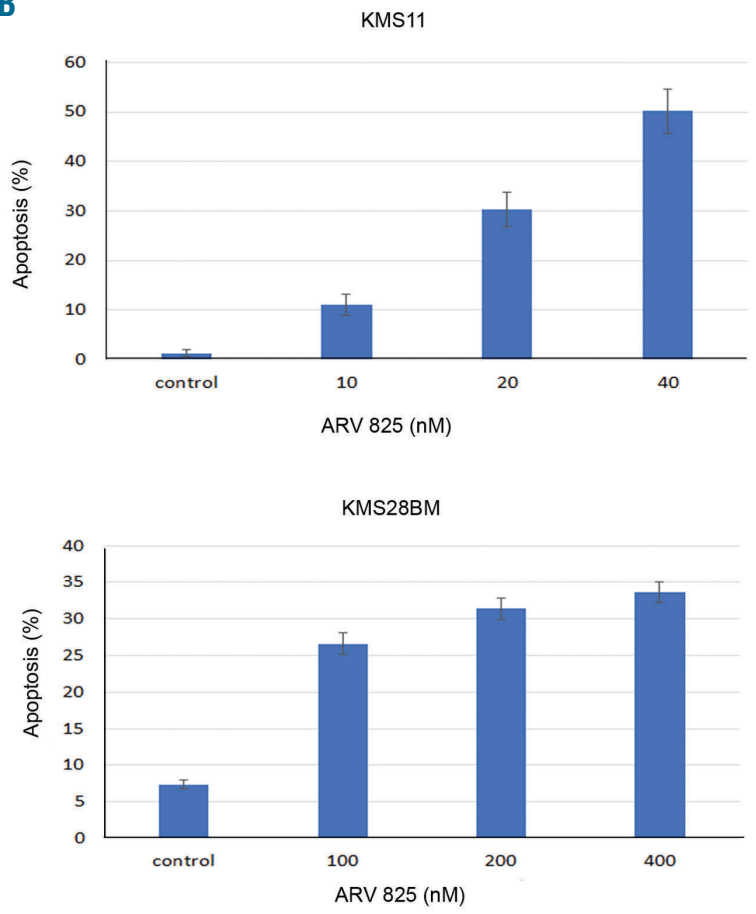

Figure 4. ARV 825 induced cell cycle arrest and apoptosis of multiple myeloma (MM) cells. (A) Cell cycle: KMS11 and KMS28BM MM cells were treated for 48 hours (h) with either ARV 825 (2.5-10 nM or 25-50 nM, respectively, $48 \mathrm{~h}$ ) or diluent control [dimethyl sulfoxide (DMSO)], stained with propidium iodide (PI) and analyzed by flow cytometry. Histograms showed proportion of cells in different phases of cell cycle. Representative of three independent experiments. (B) Apoptosis: KMS11 and KMS28BM cells treated with of ARV 825 (10-40 nM or 100-400 nM, respectively, for $48 \mathrm{~h}$ ), stained with annexin V-FITC and PI, and analyzed by flow cytometry. Histograms represent percentage of apoptotic cells. Mean \pm Standard Deviation of three independent experiments. ${ }^{*} P \leq 0.01$; $* * P \leq 0.001 ; * * * P \leq 0.0001$ for ARV 825 versus control. 
Levels of CRBN mRNA as a potential biomarker of sensitivity of multiple myeloma cells to inhibition by ARV 825

CRBN expression tends to be higher in hematologic malignancies, including $\mathrm{MM}$, compared to solid tumors (Figure 2A). Levels of CRBN mRNA expression across different $\mathrm{MM}$ cell lines positively correlated with sensitivity of the MM cells to ARV 825 (Figure 2B, left). Isogenic lenalidomide resistant cells (MM1S res and KMS11 res cells) had reduced CRBN levels and were more resistant to ARV 825 compared to their parental cells (Figure 2B, right). Over-expressed or silenced CRBN in both KMS11 and KMS28BM MM cells correlated with ability of ARV 825 to either enhance (Figure 2C) or to decrease (Figure 2D) the inhibition of cell growth, respectively.

A Combined effect of VEGFR inhibitor (Cediranib) with ARV 825
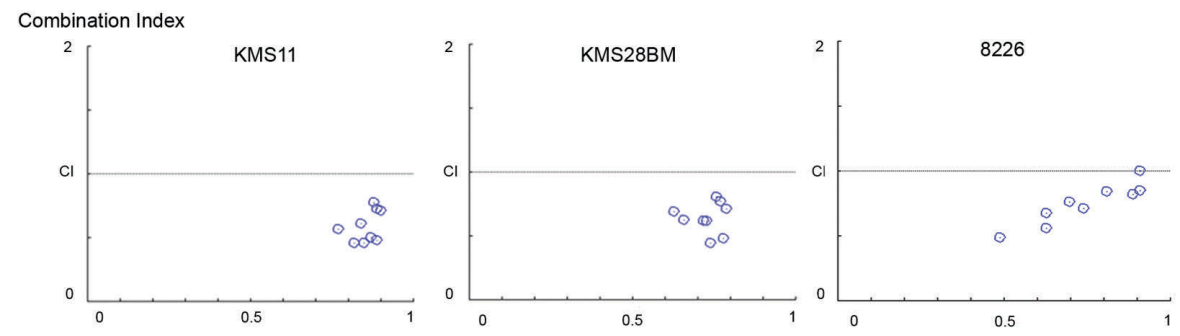

B

Combined effect of PDGFR $/ \beta$ and FLT3 inhibitors (Crenolanib) with ARV 825

Combination Index

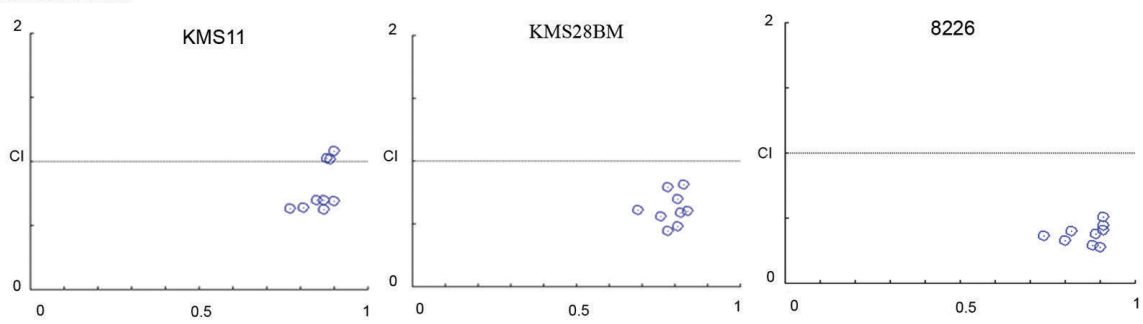

C

Combined effect of IGF-1R inhibitor (GSK1904529A) with ARV 825

Combination Index

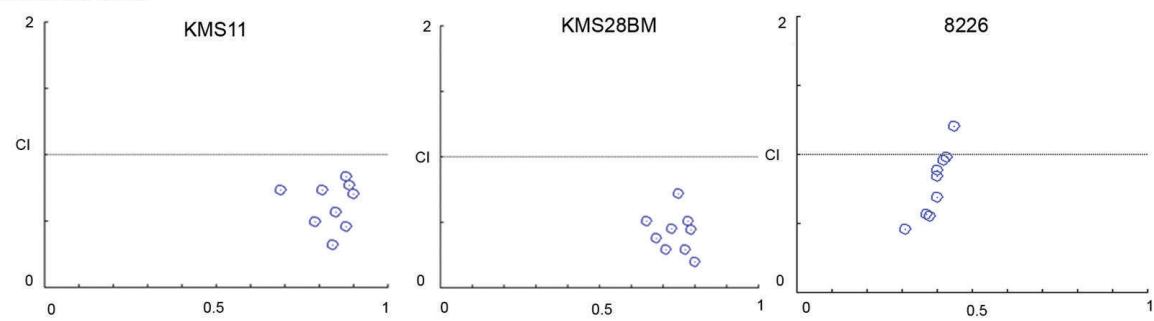

D Combined effect of VEGFR1/2/3 inhibitor (Motesanib) with ARV 825

Combination Index
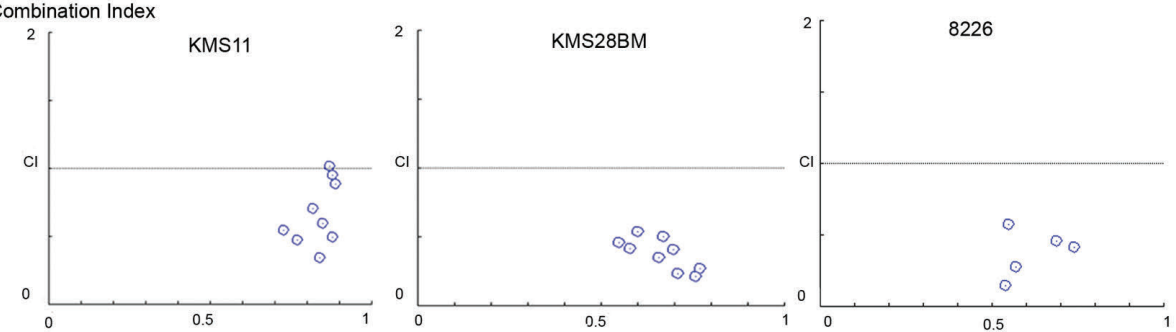

E

Combined effect of PI3K/mTOR inhibitor (LY3023414) with ARV 825 Combination Index

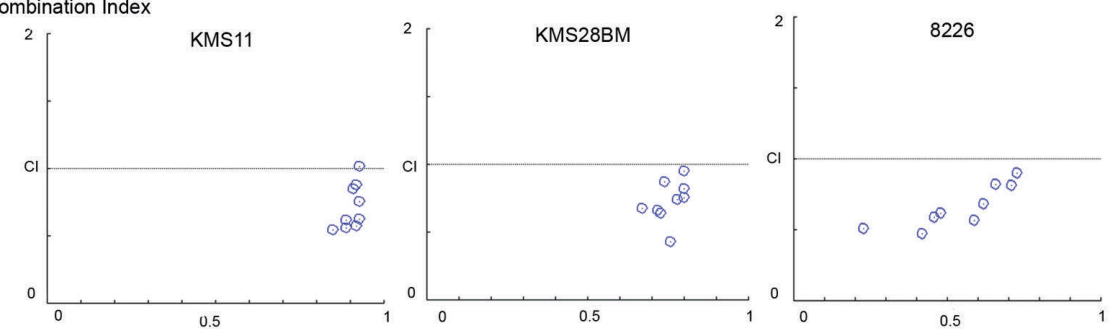

Figure 5. Combination Index (Cl) plot of ARV 825 with small molecule inhibitors. Synergistic growth inhibition (MTT assay) of KMS11 and KMS28BM multiple myeloma cells in the presence of ARV 825 and a small molecule inhibitor. $\mathrm{Cl}$ defines interaction between ARV 825 and small molecule inhibitor as plotted against a fraction of cell viability. $\mathrm{Cl}<1, \mathrm{Cl}=1$, and $\mathrm{Cl}>1$ represent synergism, additive, and antagonism of the two compounds, respectively. Values of $\mathrm{Cl}$ analysis are shown in Online Supplementary Table S3. 
ARV 825 degrades bromodomain extraterminal domain proteins

We evaluated the ability of ARV 825 to degrade BRD 2, $\mathrm{BRD} 3$ and BRD 4 proteins after KMS11 and KMS28BM cells were treated with $10 \mathrm{nM}$ and $100 \mathrm{nM}$ ARV 825, respectively, for either $2,8,24$ or $48 \mathrm{~h}$. In addition, after treatment for 48 h, ARV 825 was washed out and BRD 2, $\mathrm{BRD} 3$ and $\mathrm{BRD} 4$ proteins were examined at $6 \mathrm{~h}, 24 \mathrm{~h}$ and $48 \mathrm{~h}$ post wash-out (Figure 3A). Lysates were analyzed by western blot. ARV 825 significantly degraded BRD 2 and BRD 4 by $2 \mathrm{~h}$, but the effect on levels of BRD 3 protein was minimal. After wash-out in KMS11, BRD 2 returned

A Combined effect of VEGFR inhibitor (Cediranib) with MZ1

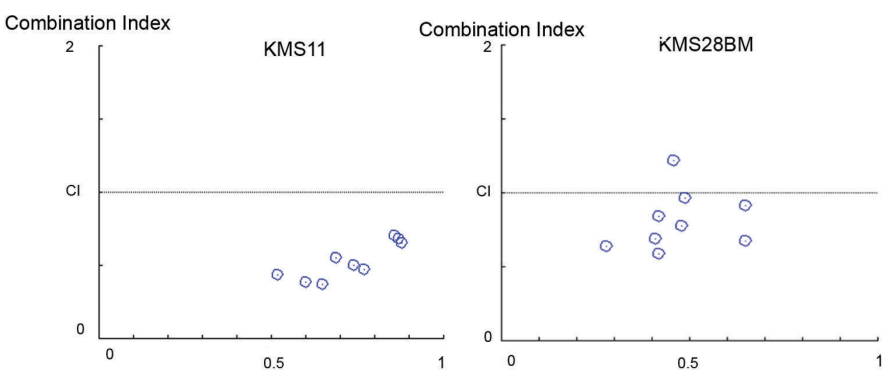

B Combined effect of PDGFR $\alpha / \beta$ and FLT3 inhibitor (Crenolanib) with MZ1

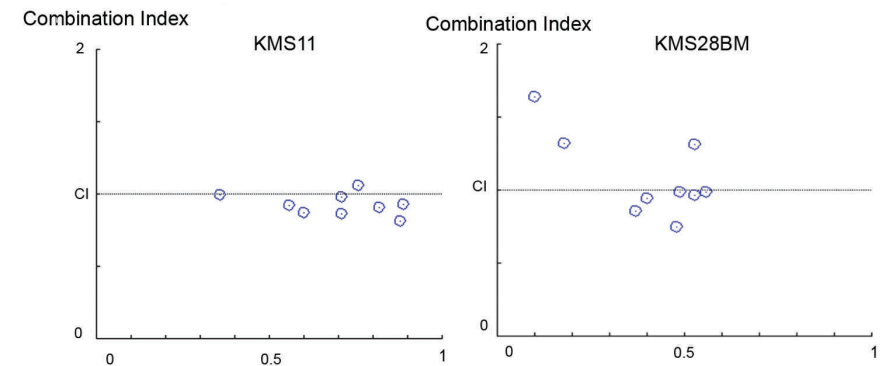

C Combined effect of IGF-1R inhibitor (GSK1904529A) with MZ1

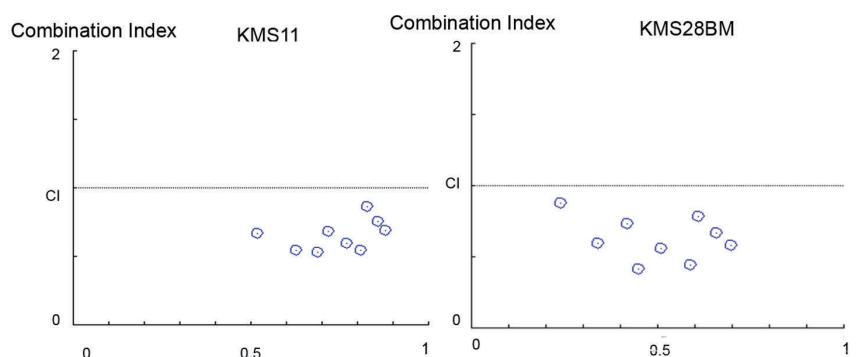

D Combined effect of VEGFR1/2/3 inhibitor (Motesanib) with MZ1

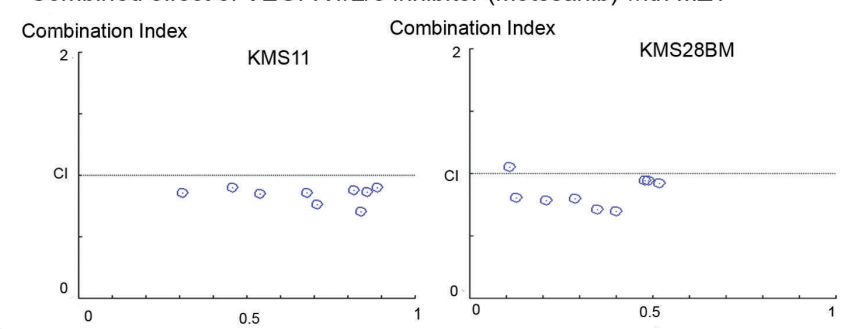

E Combined effect of PI3K/mTOR inhibitor (LY3023414) with MZ1

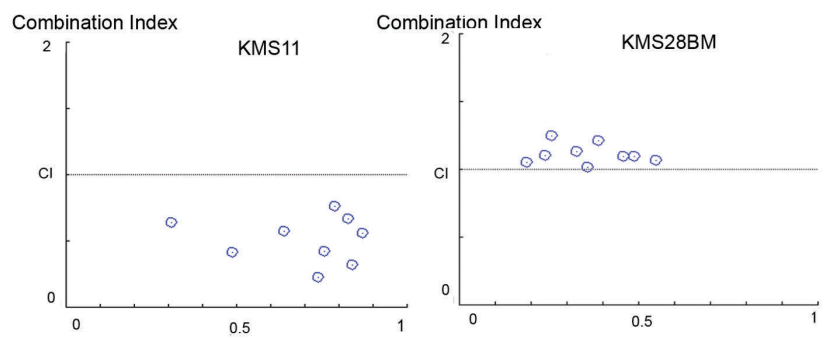

Figure 6. Combination Index (Cl) plot of MZ1 with small molecule inhibitors. Synergistic growth inhibition of KMS11 and KMS28BM multiple myeloma cells in the presence of MZ1 and small molecule inhibitors as measured by MTT assay. $\mathrm{Cl}$ defines interaction between MZ1 and small molecule inhibitor as plotted against a fraction of cell viability. $\mathrm{Cl}<1, \mathrm{Cl}=1$, and $\mathrm{Cl}>1$ represent synergism, additive, and antagonism of the two compounds, respectively. Values of $\mathrm{Cl}$ analysis are shown in Online Supplementary Table S4. 
to baseline by $24 \mathrm{~h}$ and BRD 4 was still less than control at $48 \mathrm{~h}$. For KMS28BM, levels returned to baseline for BRD 2 and BRD 4 by 6 and 24 h, respectively.

\section{ARV 825 PROTAC: CRBN expression levels correlated} with levels of degradation of bromodomains 4 and MYC

We examined whether CRBN expression correlated with effect of ARV 825-mediated degradation of BRD 4 and MYC levels. The KMS11 and KMS28BM cells+over- expressed CRBN were treated with $10 \mathrm{nM}$ and $100 \mathrm{nM}$ ARV 825, respectively, for $4 \mathrm{~h}$. Levels of BRD 4 and MYC RNA and protein were measured. CRBN over-expressed KMS11 and KMS28BM cells markedly decreased their RNA and protein levels of BRD 4 and MYC compared to their wild-type cells after ARV 825 treatment (Figure 3B) (KMS11 cells showed a greater decrease in BRD 4 and MYC compared to KMS28BM cells exposed to ARV 825).
A

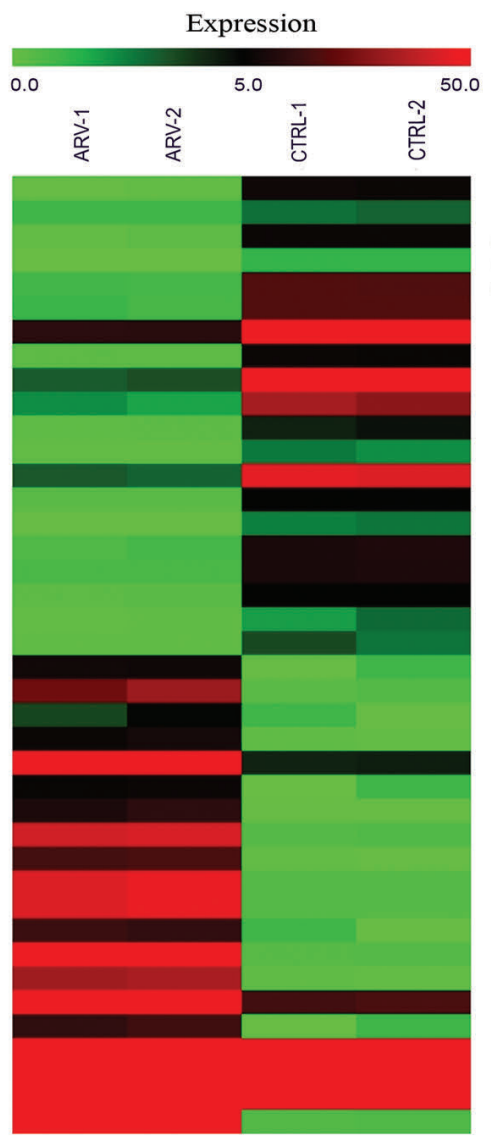

Figure 7. RNA sequencing to obtain profile of gene expression. (A) Heatmaps show top 20 down- and up-regulated genes upon ARV 825 treatment [ $20 \mathrm{nM}$ ARV 825,8 hours (h)] of KMS11 MM cells. Results of two replicates from each group are shown. (B) Level of mRNA of nine genes were validated by quantitative realt-time polymerase chain reaction. (These genes were chosen because their expression levels were altered as viewed by RNA-sequencing). Expression of each gene was normalized to $\beta$-actin as a reference (control value converted to the value of 1 ). ${ }^{*} P \leq 0.01 ; * * P \leq 0.001 ; * * * P \leq 0.0001$.

B
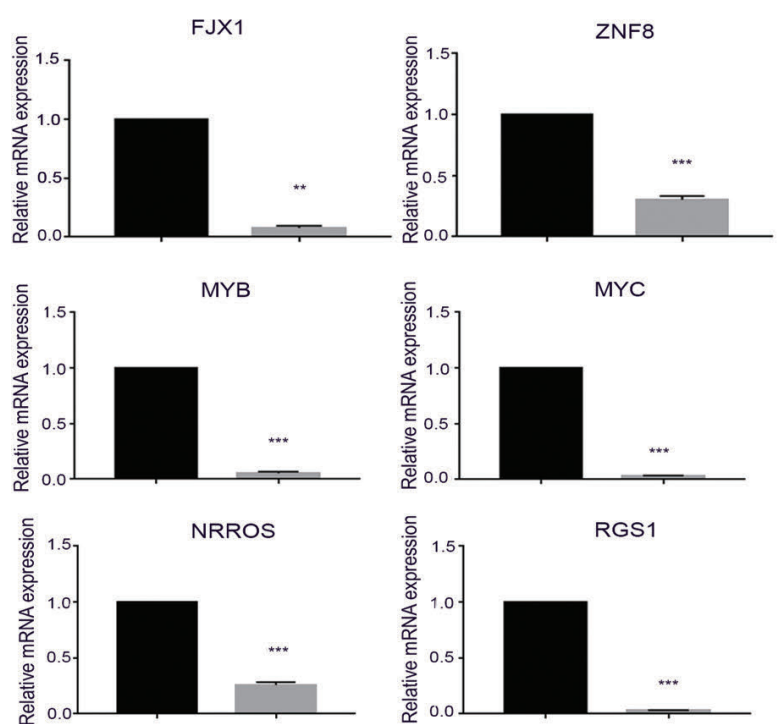
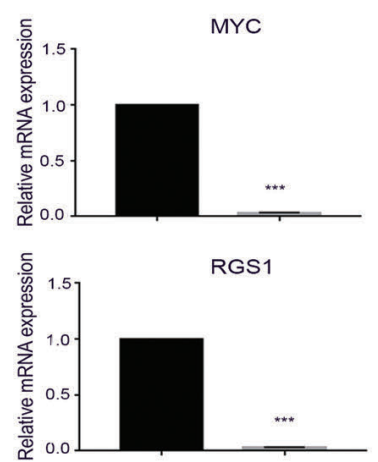

ZNF8

$\mathrm{KCNJ} 12$

MYB

NRROS

RGS1

STAMBPL

CLDN1

COA7

FDXACB1

RAB3IL1

CCDC102A

HPDL

ZEB2

ATXN7L2

EFCAB8

ADAMTSL4-AS

F13B

HFE

HIST2H2AA3

CEACAMP 3

CETP

GPR110

SERPINC1

HIST2H3A

HIST2H3A

FCGR1A

PKNOX2

ADAMTSL4

MIR663B

NSG2

MIR663A

MIR3687

MIR3648

LINC00273

(1)




\section{Degradation of IKZF 1/3 after exposure to ARV 825}

The effect of ARV 825 on IKZF $1 / 3$ degradation was examined using KMS11 and KMS28BM cells [12 h ARV 825 (10 nM, KMS11; 100 nM, KMS28BM), MZ1 (100 nM, KMS11; $70 \mathrm{nM}$, KMS28BM), pomalidomide (10 $\mu \mathrm{M})$, bortezomib (5 $\mathrm{nM}$ ) and combination of PROTAC with either pomalidomide and bortezomib] ARV 825, but not MZ1, degraded IKZF 1/3 (Ikaros/Aiolos), although the degradation was not as significant as was pomalidomide $(10 \mu \mathrm{M})$ alone. In contrast, BRD 4 was prominently degraded by both PROTAC (Figure 3C).
Bortezomib and pomalidomide antagonized the activity of ARV 825

Pomalidomide reversed the BRD 4 degradation induced by ARV 825, but not by MZ1 (Figure 3C). Pomalidomide was antagonistic to ARV 825 (Figure 3D). Pomalidomide competed with ARV 825 for the binding to CRBN; but, as expected, MZ1 activity was not influenced by pomalidomide. In contrast, the proteasome inhibitor (bortezomib) antagonized the ability of PROTAC to degrade BRD 4 (Figure $3 \mathrm{C}$ and $\mathrm{D}$ ), indicating the need for an intact proteasome function for PROTAC action. These findings are consistent with a previous study by Zhang et al. ${ }^{13}$
A

Vehicle

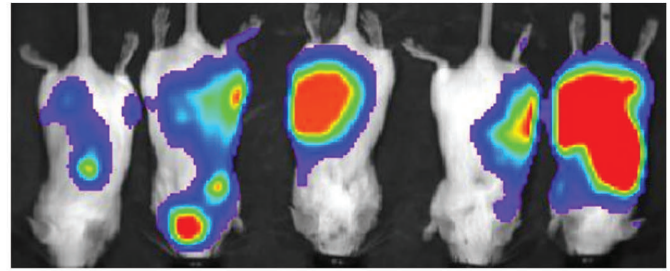

ARV 825

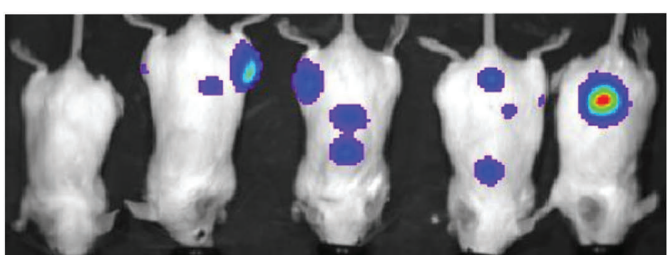

B

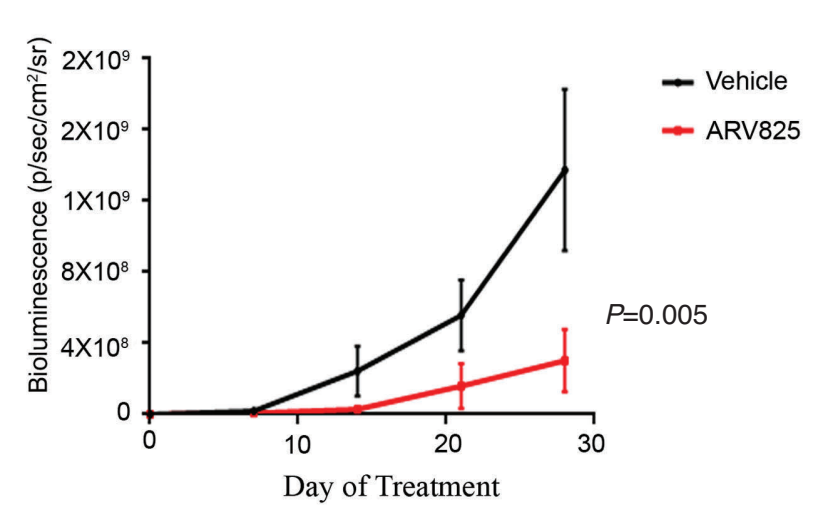

C
$\mathrm{X} 10^{8}$

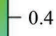

$-0.2$

Radiance (p/sec/cm²/sr)

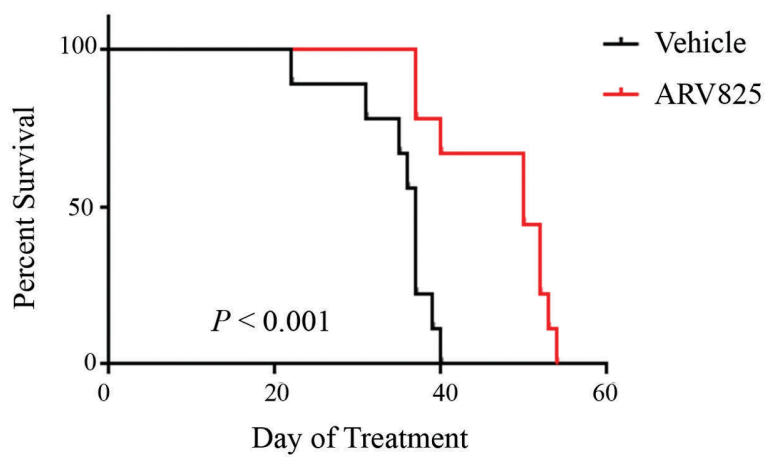

Figure 8. ARV 825 inhibits multiple myeloma (MM) samples in vivo. (A) Whole-body bioluminescence images of SCID-beige mice after intravenous injection with KMS11 ${ }^{\text {LuC }}$ cells ( 14 days) followed by treatment with ARV 825 (5 mg/kg intraperitoneally daily for 28 days) versus vehicle control. (B) Tumor burden as measured by bioluminescence in SCID-beige mice after intravenous injection with KMS11 ${ }^{\text {Lu }}$ cells (graphic display). Data represent Mean \pm Standard Deviation ( $n=9$ per group). (C) Survival curves (Kaplan-Meier) of immunodeficient mice who received human MM. The mice who received ARV 825 statistically lived longer (log-rank test, $P<0.001$ ). 


\section{ARV 825 induces cell cycle arrest and apoptosis in} multiple myeloma cells

Cell cycle analysis of MM cells was performed in the presence of various concentrations of ARV 825 for $48 \mathrm{~h}$ compared to control cells. The drug increased the G1 phase and decreased the $S$ and G2/M phases in MM cells (Figure 4A). Flow cytometric analysis of KMS11 and KMS28BM MM cells showed a marked dose-dependent increase in the percentage of apoptotic cells (apoptotic cells defined as Annexin $\mathrm{V}^{+}$and $\mathrm{PI}^{+}$) after treatment with various concentrations of ARV 825 for $48 \mathrm{~h}$ (Figure 4B). For example, ARV 825 (10, 20 and $40 \mathrm{nM}$ ) led to $10 \%, 30 \%$ and $50 \%$ of apoptotic KMS11 cells, respectively. ARV 825 at 100,200 and $400 \mathrm{nM}$ produced $26 \%, 31 \%$ and $34 \%$, respectively, of apoptotic KMS28BM cells.

\section{Small molecule inhibitors which are synergistic with ARV 825}

We performed high-throughput small-molecule inhibitor screen (panel of 170 drugs, FDA-approved or in clinical trial) to identify novel anti-MM compounds that may have synergistic activity with ARV 825. IC50s were determined for each compound, both alone and in combination with ARV 825. ARV 825 relatively sensitive KMS11 and relatively resistant KMS28BM MM cells were examined. Of the 170 tested drugs, 60 are shown in Online Supplementary Figure S3A. Combination of ARV 825 and cediranib (VEGFR inhibitor), crenolanib (PDGFR $\alpha / \beta$ and FLT3 inhibitor), GSK 1904529A (IGF-1R inhibitor), motesanib (VEGFR1/2/3 inhibitor), and LY3023414 (dual PI3K/mTOR inhibitor) produced synergistic growth inhibitory activity against both KMS11 and KMS28BM. Additional confirmation of synergistic effect of these five promising small molecules on 8226 cells was performed [Combination Index (CI) <1] (Figure 5). Selinexor (CRM1 inhibitor), gilteritinib (FLT3/AXL inhibitor), LY333531 (PKC 31 and 2 inhibitor), IGC003 (CBP/EP300 inhibitor), ruxolitinib (JAK inhibitor) produced synergistic growth inhibitory activity against either KMS11 or KMS28BM cells (Online Supplementary Figure S3B). The CI analysis of 10 of these synergistic small molecule inhibitors with ARV 825 is shown in Online Supplementary Table S3.

We also performed combination of MZ1 with promising small molecules (cediranib, Crenolanib, GSK 1904529A, motesanib and LY3023414). Each combination showed synergistic activity with MZ1 inhibiting growth of both KMS11 and KMS28BM MM cells. However, combination of MZ1 with LY3023414 has only synergistic effect against KMS11 but not KMS28BM cells (Figure 6). The CI analysis of these promising small molecule inhibitors with MZ1 is shown in Online Supplementary Table S4. A list of inhibitors is provided in Online Supplementary Table S5.

\section{Transcriptome analysis showed MYC is significantly} down-regulated by ARV 825 in multiple myeloma cells

We examined the effect of ARV 825 on mRNA expression of MM cells (KMS11) by RNA sequencing. Heatmaps (Figure 7A) displayed the top 20 down-regulated and upregulated transcripts of KMS11 MM cells following treatment of the cells with 20 nM ARV 825 for 8 h. ARV 825 markedly down-regulated CCR1, RGS1, MYB, and MYC. RNA sequencing data were further verified using quantitative RT-PCR for 9 selected genes (FJX1, ZNF8, SSTR3, CCR1, MYB, NRROS, MYC, RGS1 and DOK4) in KMS11
(Figure 7B) and KMS28BM cells (Online Supplementary Figure S2A). Furthermore, Gene-Set Enrichment Analysis (GSEA) indicated the robust downregulation of functionally-defined MYC targets following ARV 825 treatment of KMS11 MM cells (Online Supplementary Figure S2B and C). Primers for qRT-PCR are listed in Online Supplementary Table S6.

\section{ARV 825 inhibited multiple myeloma growth in vivo}

Anti-proliferative effect of ARV 825 was examined in vivo against $\mathrm{MM}$ xenografts growing in SCID-Beige mice. Two weeks after injection, the MM cells were easily observed by bioluminescence imaging, after which, mice ( $n=9$ per group) were randomly assigned to receive either ARV $825(5 \mathrm{mg} / \mathrm{kg})$ dissolved in $200 \mu \mathrm{L}$ of vehicle daily intraperitoneally or $200 \mu \mathrm{L}$ of vehicle alone. ARV 825 significantly slowed tumor growth in experimental mice compared to control mice receiving vehicle, as measured by bioluminescence (Figure 8A and B) at days 7, 14, 21 and 28. Importantly, ARV 825 treatment significantly prolonged the murine OS compared to vehicle-treated mice (Figure 8C). ARV 825 treated mice maintained normal activity and insignificant weight loss compared to diluent control mice (Online Supplementary Figure S4A). The IC50 of ARV 825 using normal mouse BM cells $\left(2 \times 10^{5}\right.$ cells/well) is $500 \mathrm{nM}$ (Online Supplementary Figure S4B).

\section{Discussion}

Despite the major advances in the treatment of MM made over the last decade, disease management still remains challenging as most patients either do not achieve a complete remission or eventually relapse. Bortezomib and lenalidomide have become a part of standard management. Auto-transplants are often also given; nevertheless, patients are rarely cured. New targeted therapeutic strategies are needed. Next generation BET inhibitor ARV 825 degrades bromodomains. We found BRD 2 and BRD 4 were profoundly depleted, consistent with previous reports of PROTAC for other malignancies. ${ }^{7,9,10,16}$ Previous studies have shown a fusion of JO1 and thalidomide (dBET6) has significant potency against $\mathrm{MM} .{ }^{17} \mathrm{We}$ also showed that ARV 825 leads to significant growth inhibition of myeloma cells in liquid culture, clonogenic assay and, most importantly, in a xenograft model. Flow cytometric analysis showed that ARV 825 induced apoptosis and G0/G1 cell cycle arrest of these cells in vitro.

We demonstrated that both ARV 825 and MZ1 have promising activity against MM cells. ARV 825 induced degradation of BET proteins via CRBN E3 ligase. Importantly, we found a positive correlation of intracellular levels of CRBN and their sensitivity to ARV 825. CRBN expression is prominent in hematologic malignancies, including MM. Response to immunomodulatory drugs is clinically correlated with expression of CRBN ${ }^{18}$ Loss of function of CRBN causes resistance to dBET6 by perturbing dBET-mediated BRD 4 degradation. ${ }^{17}$ We postulate that levels of CRBN will serve as a predictive biomarker for cellular responsiveness to ARV 825. Indeed, two pairs of isogenic cells, one of each pair resistant to lenalidomide (KMS11 res and MM1S res), had very low expression of CRBN. Genetically either silencing or over-expressing CRBN decreased and increased, respectively, the sensitivity of an MM cell line to growth inhibition by ARV 825. In 
stark contrast, their sensitivity to MZ1, which uses VHL E3 ligase, remained unchanged after either forced expression or silencing CRBN. Taken together, data suggest that ARV 825 may be most potent when this PROTAC is given to patients whose MM cells express CRBN. The sensitivity of MM patients to ARV 825 increased as the CRBN expression increased. In contrast, MZ1 could be a promising therapeutic drug for lenalidomide/pomalidomideresistant MM. Our ARV 825 data are consistent with a recent study which demonstrated that pomalidomide competed with ARV 825 for binding to CRBN. ${ }^{13}$ The authors further showed that ARV 825 relied on an intact proteasome pathway with proteasome inhibitors (carfilzomib or bortezomib) antagonizing the effects of ARV $825 .^{13}$ This is consistent with our findings.

Based on high throughput screening of small-molecule inhibitors, we identified novel compounds that have synergistic activity with ARV 825 against MM cell lines (KMS11 and KMS28BM), including those against either dual PI3K/mTOR, VEGFR, PDGFR $\alpha / \beta$, and FLT3 and IGF1R. Previous studies showed the inhibition of PI3K and BET blocked reactivation of PI3K signaling in diverse cancer models. ${ }^{19}$ Interestingly, a previous study reported that co-treatment of ARV 825 with ruxolitinib synergistically inhibited growth of secondary acute myeloid leukemia. ${ }^{9}$ We also observed synergism of this combination against KMS28BM MM cells, but not KMS11 MM cells (Online Supplementary Figure S2B).
RNA sequencing and gene set enrichment analysis demonstrated that MYC expression is significantly downregulated after treatment with ARV 825 ( $8 \mathrm{~h}$ ). MYC is an attractive target in $\mathrm{MM}$ due to its role in disease progression. In addition, CCR1 and LGR5 were down-regulated. CCR1 has been reported to play a central role in pathogenesis of MM as well as MM-induced osteolytic bone disease,$^{20}$ whereas LGR5 has been identified as a marker of early stem cells in the intestine. ${ }^{21}$

In summary, our studies showed that MM cells are sensitive to ARV 825 and a combination of ARV 825 with synergistic small molecule inhibitors may be therapeutically effective for patients. During the final preparation of our manuscript, another manuscript was published reporting ARV 825 in MM. ${ }^{13}$ Taken together, these two studies nicely compliment each other and provide the foundation for further pre-clinical studies of both ARV 825 and MZ1 for the treatment of MM.

\section{Acknowledgments}

We thank Aaron Eshman and Melmed family for their invaluable support for this myeloma project. We also express gratitude to Morgan Stanley Inc.

\section{Funding}

This work was also supported by a grant from the Leukemia and Lymphoma Society.

\section{References}

1. Siegel RL, Miller KD, Jemal A. Cancer statistics, 2018. CA Cancer J Clin. 2018; 68(1):7-30

2. Cohen AD, Garfall AL, Stadtmauer EA, et al. B-Cell Maturation Antigen (BCMA)Specific Chimeric Antigen Receptor T Cells (CART-BCMA) for Multiple Myeloma (MM): Initial Safety and Efficacy from a Phase I Study. Blood. 2016;128(22):1147.

3. Shu S, Polyak K. BET Bromodomain Proteins as Cancer Therapeutic Targets. Cold Spring Harb Symp Quant Biol. 2016;81:123-129.

4. Mochizuki K, Nishiyama A, Jang MK, et al. The Bromodomain Protein Brd4 Stimulates G 1 Gene Transcription and Promotes Progression to S Phase. J Biol Chem. 2008;283(14):9040-9048.

5. Lovén J, Hoke HA, Lin CY, et al. Selective inhibition of tumor oncogenes by disruption of super-enhancers. Cell. 2013; 153(2):320-334.

6. Delmore JE, Issa GC, Lemieux ME, et al. BET bromodomain inhibition as a therapeutic strategy to target c-Myc. Cell. 2011;146(6):904-917

7. Lu J, Qian Y, Altieri M, et al. Hijacking the E3 Ubiquitin Ligase Cereblon to Efficiently Target BRD4. Chem Biol. 2015;22(6):755763.

8. Buckley DL, Crews CM. Small-Molecule
Control of Intracellular Protein Levels through Modulation of the Ubiquitin Proteasome System. Angew Chemie Int Ed. 2014;53(9):2312-2330.

9. Saenz DT, Fiskus W, Qian Y, et al. Novel BET protein proteolysis-Targeting chimera exerts superior lethal activity than bromodomain inhibitor (BETi) against post-myeloproliferative neoplasm secondary (s) AML cells. Leukemia. 2017;31(9):1951-1961.

10. Sun B, Fiskus W, Qian Y, et al. BET protein proteolysis targeting chimera (PROTAC) exerts potent lethal activity against mantle cell lymphoma cells. Leukemia. 2018; 32(2):343-352.

11. Raina K, Lu J, Oian Y, et al. PROTACinduced BET protein degradation as a therapy for castration-resistant prostate cancer. Proc Natl Acad Sci U S A. 2016; 113(26):7124-7129

12. Winter GE, Buckley DL, Paulk J, et al. DRUG DEVELOPMENT. Phthalimide conjugation as a strategy for in vivo target protein degradation. Science. 2015;348 (6241):1376-1381.

13. Zhang X, Lee HC, Shirazi F, et al. Protein targeting chimeric molecules specific for bromodomain and extra-terminal motif family proteins are active against pre-clinical models of multiple myeloma. Leukemia. 2018:32(10):2224-2239.

14. Zhu YX, Braggio E, Shi C-X, et al. Cereblon expression is required for the antimyeloma activity of lenalidomide and pomalido- mide. Blood. 2011;118(18):4771-4779.

15. Zhu YX, Shi C-X, Bruins LA, et al. Lenalidomide, Pomalidomide and CC-220 resistant multiple myeloma: confirmation of role of CRBN/IRF4 axis and identification of multiple disruption mechanisms. Blood. 2017:130(Suppl 1):4369.

16. Piya S, Bhattacharya S, Mu H, et al. BRD4 Proteolysis targeting chimera (PROTAC) ARV-825, causes sustained degradation of BRD4 and modulation of chemokine receptors, cell adhesion and metabolic targets in leukemia resulting in profound antileukemic effects. Blood. 2016; 128(22):748.

17. Matthews GM, Hu Y, Sheffer M, et al. Abstract 4713: BET bromodomain degradation as a therapeutic strategy in drug-resistant multiple myeloma. Cancer Res. 2016; 76(14):4713.

18. Schuster SR, Kortuem KM, Zhu YX, et al. The clinical significance of cereblon expression in multiple myeloma. Leuk Res. 2014; 38(1):23-28.

19. Stratikopoulos EE, Dendy M, Szabolcs M et al. Kinase and BET Inhibitors Together Clamp Inhibition of PI3K Signaling and Overcome Resistance to Therapy. Cancer Cell. 2015;27(6):837-851.

20. Vallet S, Anderson KC. CCR1 as a target for multiple myeloma. Expert Opin Ther Targets. 2011;15(9):1037-1047.

21. Clevers H. Searching for adult stem cells in the intestine. EMBO Mol Med. 2009; 1(5):255-259. 